\title{
Glucose Limitation Sensitizes Cancer Cells to Selenite-Induced Cytotoxic Effect via SLC7A11- Mediated Redox Collapse
}

Hui Chen

China Agricultural University

Han Zhang

China Agricultural University

Lixing Cao

China Agricultural University

Jinling Cui

China Agricultural University

Xuan Ma

China Agricultural University

Chong Zhao

China Agricultural University

Shutao Yin

China Agricultural University

Hongbo Hu ( $\square$ hongbo@cau.edu.cn )

China Agricultural University

\section{Research}

Keywords: selenite, glucose limitation, SLC7A11, NADPH, ROS

Posted Date: September 28th, 2021

DOI: https://doi.org/10.21203/rs.3.rs-934222/v1

License: (1) (1) This work is licensed under a Creative Commons Attribution 4.0 International License. Read Full License 


\section{Abstract}

Background: Combination of fasting with chemotherapy has been drawn an increasing attention because of the encouraging efficacy. SLC7A11 is frequently over-expressed in most of cancer cells, and elevated expression of SLC7A11 renders cancer cells more susceptible to glucose starvation owing to SLC7A11mediated redox collapse. Selenite is a representative inorganic form of selenium, and is preferentially accumulated in tumors. This selenophilic peculiarity of cancer cells is closely associated with the elevated expression of SLC7A11. Given the established the link among glucose deprivation, SLC7A11, oxidative stress and selenite, we hypothesized that glucose starvation could specifically sensitize cancer cells to selenite-mediated cytotoxic effect.

Methods: The cytotoxic effect of combining selenite with glucose starvation on cancer cell was assessed by crystal violet staining and Annexin V/PI staining. Flow cytometry were employed to assess intracellular ROS levels, labile iron pool and lipid peroxidation. Xenograft models were used to test its in vivo antitumor activity. Commercial assay kit, LC-MS, RNA interference and western blot were applied to investigate the mechanism underlying synergistic effect.

Results: It showed that cytotoxic effect of selenite on cancer cells, but not on normal cells, was significantly enhanced in response to the combination of selenite and glucose limitation. Furthermore, in vivo therapeutic efficacy of combining selenite with fasting was dramatically improved in xenograft models of lung and colon cancer. Mechanistically, we found that SLC7A11 expression in cancer cells was up-regulated by selenite both in vitro and in vivo. The elevated SLC7A11 led to accumulation of cystine, depletion of $\mathrm{NADPH}$, and inhibition of cystine to cysteine conversion, which in turn boosted selenitemediated reactive oxygen species (ROS), followed by enhancement of selenite-mediated cytotoxic effect.

Conclusion: The findings of the present study provide an effective and practical approach for increasing the therapeutic window of selenite, and imply that combination of selenite with fasting holds promising potential to be developed a clinically useful regimen for treating certain types of cancer.

\section{Background}

Combination of intermittent fasting (IF)/fasting-mimicking diet (FMD) with chemotherapy has been drawn an increasing attention. Accumulating evidence indicates that this combination treatment can not only increase cancer therapeutic effect, but also reduce detrimental effects of chemotherapy on normal cells[1-4]. Therefore, IF/FMD is proposed as a promising strategy to improve therapeutic efficacy and prevent side effects. This differential effect on cancer and normal cells is attributed to the different adaptability of cancer and normal cells to the starvation condition[5]. Cancer cells are more vulnerable to the starvation condition partially due to its aberrant metabolic attributes. For example, the Warburg effect is a key feature of cancer cell metabolism. Shortage in the availability of glucose by fasting forces a shift from aerobic glycolysis (Warburg effect) to mitochondrial oxidative phosphorylation in cancer cells to meet the requirement for cancer cell growth[1,6]. The increased oxidative phosphorylation results in 
elevated ROS generation, which in turn renders cancer cells susceptible to the chemotherapy[7, 8]. A recent study by Liu et al[9] revealed the involvement of SLC7A11 in vulnerability of cancer cells to glucose starvation. SLC7A11, a cystine/glutamate antiporter, is responsible for uptake of extracellular cysteine to maintain cellular redox balance. SLC7A11 is overexpressed in many types of cancers including lung cancer and triple-negative breast cancer[10,11]. Overexpression of SLC7A11 promotes tumor development via suppressing oxidative stress-induced ferroptosis and non-ferroptotic cell death[12, 13]. On the other hand, overexpression of SLC7A11 leads to increase of cystine uptake, accompanied by augmentation of glutamate export and NADPH consumption (due to NADPH-dependent reduction of cystine to cysteine), which forces cancer cells to be highly dependent on pentose phosphate pathway (PPP)[9]. Such metabolic feature confers the cancer cells with elevated expression of SLC7A11 more sensitive to glucose deprivation owing to cystine accumulation-mediated NADPH depletion and impairment of cellular redox homeostasis.

Selenium is an essential micronutrient with multiple biological functions. Among them, the anticancer activity has been extensively investigated and yield controversial outcomes. It is generally believed that the anticancer activity of selenium is associated with dose levels, forms and nutrient status of the body[14]. Selenite, a representative inorganic form of selenium, is preferentially accumulated in tumors $[15,16]$. Preclinical and clinical studies have shown that selenite alone or in combination with chemotherapy or radiotherapy is effective against a variety of cancer types[17-21]. Mechanistically, induction of reactive oxygen species and activation of p53 play critical role in its cytotoxic and sensitization effect[22]. Recent studies revealed that selenium enrichment in tumor is closely associated with the elevated expression of SLC7A11, supporting an important role of SLC7A11 in the selenophilic feature of cancer cells[10, 11].

As discussed above, elevated expression of SLC7A11 renders cancer cell more susceptible to glucose limitation due to redox imbalance. We hypothesized that SLC7A11 might be up-regulated in response to selenite challenge, and therefore combining selenite with glucose starvation could achieve synergistic induction of oxidative stress and cytotoxic effect on cancer cells. This hypothesis was tested in the present study using both in vitro and in vivo models.

\section{Materials And Methods}

\section{Chemicals and reagents}

Sodium selenite (Se), methylseleninic acid (MSeA) dihydroethidium (DHE), 2',7'-

dichlorodihydrofluorescein diacetate (DCFH-DA), glutamine (GIn), glucose and manganese(III) tetrakis(1methyl-4-pyridyl)porphyrin (MnTMPyP) were purchased from Sigma Chemical Co. (St. Louis, MO, U.S.A.). BAY-876, deferoxamine mesylate (DFO) and ferrostatin-1 (Fer-1) were purchased from MedChem Express (Monmouth Junction, NJ, U.S.A.). Monosodium glutamate (MSG), salicylazosulfapyridine (SAS) and 2deoxy-D-glcose (2-DG) were purchased from Aladdin (Shanghai, China). NADPH were purchased from solarbio (Beijing, China). FerroOrange and Liperfluo were purchased from DOJINDO (Kyushu, 
Japan). Antibodies specific for SLC7A11 (12691), SLC7A11 (98051) and $\beta$-actin (3700) were purchased from Cell Signaling Technology (Beverly, MA, U.S.A.). The secondary antibodies: Horseradish peroxidaselinked Goat Anti-Rabbit IgG and Horseradish peroxidase-linked Goat Anti-Mouse IgG were obtained from MBL International Corporation (Beijing, China). SLC7A11 small interfering RNA (siRNA) and nontargeting siRNA were purchased from Santa Cruz Biotechnology (Santa Cruz, CA, USA).

\section{Cell culture and treatments}

HCT116 human colon cancer cells, LLC lung cancer cells, HepG2 liver cancer cells, MCF-7 and MDA-MB231 breast cancer cells, HK2 normal kidney cells and MRC-5 normal lung cells were grown in Dulbecco' modified Eagle's medium (DMEM) supplemented with $10 \%$ fetal bovine serum and $2 \mathrm{mM}$ L-glutamine unless otherwise indicated. A549 lung cancer cells, DU145 prostate cancer cells were grown in RPMI medium supplemented with $10 \%$ fetal bovine serum and $2 \mathrm{mM}$ L-glutamine unless otherwise indicated. For starvation experiments, cells were washed three times with PBS pH 7.2 and then incubated in the indicated starvation conditions. All cultures were maintained in a humidified tissue culture incubator at $37^{\circ} \mathrm{C}$ in $5 \% \mathrm{CO}_{2}$.

\section{Crystal violet staining}

After treatment with agents, the culture medium was aspirated and replaced with $1 \%$ glutaraldehyde solution for $15 \mathrm{~min}$. Then, the cells were stained with a $0.02 \%$ crystal violet solution for $30 \mathrm{~min}$. After that, the solution was replaced by $70 \%$ ethanol for solubilization. The OD value at $570 \mathrm{~nm}$ was measured by microplate reader.

\section{Cell death evaluation}

Collected cells were incubated with a fluorescein isothiocyanate-labeled annexin V (FITC) and propidium iodide (PI) for flow cytometry analyses. An Annexin V/PI staining kit (MBL International Corporation) was used.

\section{Determination of cell ROS}

Reactive oxygen species (ROS) measurement using DHE and DCFH-DA staining was performed as described as follows: cells were trypsinized, washed with PBS, and then resuspended in the medium without FBS and loaded with $10 \mu \mathrm{M}$ DHE or $20 \mu \mathrm{M}$ DCFH-DA for $30 \mathrm{~min}$ at $37^{\circ} \mathrm{C}$, incubated in the dark. Afterward, cells were washed with PBS and resuspended in the PBS used for fluorescence analysis by flow cytometer. Fluorescence increase was estimated utilizing the wavelengths $535 \mathrm{~nm}$ (excitation) and $610 \mathrm{~nm}$ (emission) for DHE, and wavelengths $485 \mathrm{~nm}$ (excitation) and $535 \mathrm{~nm}$ (emission) for DCFH-DA.

\section{GSH, NADP+, NADPH and cystetine measurement}

Glutathione (GSH) in cell was measured using a commercial kit from Nanjing Jiancheng Bioengineering Institute (Nanjing, China) following the manufacturer's instruction. The intracellular levels of NADPH and 
total NADP (NADPH+NADP ${ }^{+}$) were measured according to the protocol of manufacturer from DOJINDO (Kyushu, Japan). The quantification of cysteine was determined using Cysteine assay kit (Abcam, Cambridge, United Kingdom).

\section{Cystine measurement by LC-MS}

Cells were seeded on $75 \mathrm{~cm}$ culture flasks at a density sufficient to ensure approximately $80 \%$ confluence at the time of extraction. Cells were trypsinized, washed twice with ice cold PBS, extracted by adding 0.3 $\mathrm{mL}$ of an $50 \%$ methanol: $50 \%$ water mixture $(\mathrm{v} / \mathrm{v})$ at ice temperature and then disrupted by ultrasound. Cell debris was pelleted by centrifugation at $12000 \mathrm{rpm}$ for $10 \mathrm{~min}$ at $4^{\circ} \mathrm{C}$ and the supernatant was transferred to a fresh tube. $20 \mu \mathrm{L}$ cell extracts, $5 \mu \mathrm{L}$ internal standard substance (canocinal amino acid mix with $1.23 \mathrm{mM} \mathrm{L}$-Cystine- ${ }^{13} \mathrm{C}_{2}{ }^{-15} \mathrm{~N}_{1}$, Cambridge Isotope Laboratories) and $40 \mu \mathrm{L}$ isopropyl alcoholformic acid $\llbracket \mathrm{v} / \mathrm{v}=99: 1$ ॠwere mixtured and vortex oscillated for $2 \mathrm{~min}$, then centrifugated at $12000 \mathrm{rpm}$ for $10 \mathrm{~min}$ at $4^{\circ} \mathrm{C} .10 \mu \mathrm{L}$ supernatant were derivatized according to the protocol of AccQ Tag kit (Waters, USA) and then analyzed by LC-MS. Analyte concentrations were quantified by comparison to standard curves of cystine prepared by the same method. To determine intracellular concentrations, the concentrations of cell protein in the same volume of cell resuspension were determined.

LC-MS analysis was performed as follows: the LC-MS system consists of a Waters UPLC I-Class system and Waters XEVO TQ-XS quadrupole rods tandem mass spectrometer equipped with a ESI probe. Mass data acquisition and remote control of the LC-MS system were done by Masslynx software.

Chromatography was performed with a waters UPLC HSS T3 $(150 \times 2.1 \mathrm{~mm}, 1.8 \mu \mathrm{m}$ particle size $)$. The mobile phase consisted of solvent $A(0.1 \%$ formic , water) and solvent $B$ (acetonitrile and water, $v / v=95: 5)$ with a gradient elution (0-0.5 min, 96-96\% A, 0.5-2.5 min, 96-90\% A, 2.5-5 min, 90-72\% A, 5-6 min, 72-5\% A, 6-7 min, 5-5\% A, 7- 7.1 min, 5-96\% A, 7.1- 9 min, 96-96\% A). The flow rate of the mobile phase was 0.5 $\mathrm{mL} / \mathrm{min}$. The column temperature was maintained at $50^{\circ} \mathrm{C}$. The injection volume was $5 \mu \mathrm{L}$. The exactive was operated in positive ionization mode with an electrospray ionizatio interface. The instrument parameters were as follows: positive ESI source temperature, $50^{\circ} \mathrm{C}$, capillary voltage, $1.5 \mathrm{kV}$, cone voltage, $20 \mathrm{~V}$, cone gas flow, $150 \mathrm{~L} / \mathrm{Hr}$, desolvation gas flow, $1000 \mathrm{~L} / \mathrm{Hr}$.

\section{LPO, LIP measurement}

The total cellular labile iron pool (LIP) and lipid peroxidation (LPO) measurement using FerroOrange and Liperfluo were performed as described as follows: cells were trypsinized, washed with PBS, and then resuspended in the PBS and loaded with $1 \mu \mathrm{M}$ FerroOrange and or $1 \mu \mathrm{M}$ Liperfluo for $30 \mathrm{~min}$ at $37^{\circ} \mathrm{C}$, incubated in the dark. Then the cells were analyzed for fluorescence by flow cytometer. Fluorescence increase was estimated utilizing the wavelengths $561 \mathrm{~nm}$ (excitation) and $593 \mathrm{~nm}$ (emission) for FerroOrange, and the wavelengths $488 \mathrm{~nm}$ (excitation) and $535 \mathrm{~nm}$ (emission) for Liperfluo.

\section{RNA interference}


The cells were transfected with $40 \mathrm{nM}$ SLC7A11 siRNA or nontargeting siRNA using the INTERFERin siRNA transfection reagent according to the manufacturer's instructions (Polyplus-Transfection, Inc., New York, NY, USA) and then were used for subsequent experiments.

\section{Western blotting}

Western blotting was performed according to the method of Yan et al[23] with minor modifications. The cell lysate was prepared in ice-cold radioimmunoprecipitation assay (RIPA) buffer. Cell lysates were resolved by electrophoresis and transferred to a polyvinylidene fluoride (PVDF) membrane (Millipore, Billerica, MA, USA, IPVH00010). The blot was then probed with primary antibody followed by incubation with the appropriate horseradish peroxidase-conjugated secondary antibodies. The signal was visualized by enhanced chemiluminescence (Fisher/Pierce, Rockford, IL, USA, 32106) and recorded on an X-ray film (Eastman Kodak Company, Rochester, NY, USA, XBT-1).

\section{Xenograft tumor models}

The animals were housed under specific pathogen-free conditions at $22 \pm 2{ }^{\circ} \mathrm{C}$ with $55 \pm 10 \%$ relative humidity and with $12 \mathrm{~h}$ day/light cycles. All experiments were performed in accordance with the guidelines established in the Principles of China Agricultural University Institutional Animal Care and Use Committee. For xenograft experiments, 6-8-week-old male C57 BL/6N mice from Charles River (Beijing, China) were subcutaneously injected with $2 \times 10^{6}$ LLC cells resuspended in $100 \mu \mathrm{L}$ of PBS, 5 week-old male BALB/c nude mice from Charles River (Beijing, China) were subcutaneously injected into the dorsal side with $3 \times 10^{6}$ HCT 116 cells resuspended in $100 \mu \mathrm{L}$ of PBS. When tumors were palpable ( 5 days after inoculation), mice were randomly divided into different experimental groups. Mice were kept on the feeding/fasting protocols performed as described as the reported[1]. In short, fasting cycles were achieved by complete removal of food while allowing free access to water for $24 \mathrm{~h}$ from $6 \mathrm{pm}$ to $6 \mathrm{pm}$ of the following day when food was re-supplied ad libitum. Selenite dissolved in water at the dose of 2 $\mathrm{mg} / \mathrm{kg}$ body weight was administered every $48 \mathrm{~h}$ at 9 am (time in fasting cycle) via oral gavage. Body weights were recorded every 2 days, and tumor volumes were measured every 2 days by a digital vernier caliper according to the following equation: tumor volume $\left(\mathrm{mm}^{3}\right)=\left(\right.$ length $\times$ width $\left.{ }^{2}\right) \times 0.5$, where the length and width were expressed in millimeters.

\section{Statistical analysis}

Data were presented as the mean $\pm S D$. These data were analyzed by ANOVA with appropriate post hoc comparisons among means. $\mathrm{P}<0.05$ was considered statistically significant.

\section{Results}

\section{Glucose limitation sensitizes cancer cells to selenite-mediated cytotoxic effect}


To investigate influences of glucose on the anticancer activity of selenite, HCT116 human colon cancer cells were cultured in the medium with different levels of glucose and exposed to various concentrations of selenite $(0,1,2.5$ and $5 \mu \mathrm{M})$ for $24 \mathrm{~h}$, and the changes of cell viability were measured by crystal violet staining. As shown in Fig. 1A, selenite-mediated cytotoxic effect was dramatically enhanced when the concentration of glucose decreased to $2.5 \mathrm{mM}$. This enhancement was further validated by measurement of cell death induction using Annexin V/PI staining, and results were shown in Fig. 1B. Consistent with the cell viability changes, cell death induction by selenite in HCT116 cells was significantly increased in the context of glucose deprivation (refers to $2.5 \mathrm{mM}$ ). Glucose is primarily taken up by cancer cells via GLUT1, a member of glucose transporter family and commonly overexpressed in cancer cells. Inhibition of GLUT1 by its inhibitor is supposed to cause intracellular glucose reduction. We next examined effect of GLUT1 inhibitor BAY-876 on selenite-induced cytotoxicity of HCT116 cells, and results demonstrated that an enhanced cytotoxicity was achieved by all the combinations of selenite and BAY-876, further supporting the sensitization effect of glucose deprivation on selenite-induced cytotoxic effect against HCT116 colon cancer cells (Fig. 1C). To determine whether the sensitization effect of glucose limitation on selenite-induced cytotoxicity was specific for HCT116 colon cancer cells, LLC and A549 lung cancer cells, HepG2 liver cancer cells, MCF-7 and MDA-MB-231 breast cancer cells, and DU145 prostate cancer cells were tested. As shown in Fig. 1D, the sensitization effect was also found in all additional cancer cell lines tested, indicating general applicability of the sensitization effect. To determine if the sensitization effect was restricted to cancer cells, MRC-5 normal lung cells and HK-2 normal kidney cells were tested, and results showed that the sensitization effect was not observed in these two normal cell lines, suggesting that glucose starvation specifically potentiated cancer cells but not normal cells to selenitemediated cytotoxicity. The anticancer activity of selenium is closely associated with its forms. We asked if the sensitization effect of glucose limitation on selenite can be also achieved with other forms of selenium compounds. Influence of glucose limitation on the cytotoxicity of methylseleninic acid (MSeA), a representative of organic selenium compounds, was assessed, and results showed that glucose starvation failed to potentiate HCT116 colon cancer cells to MSeA-mediated cytotoxic effect (Fig. 1E), suggesting that the sensitization effect of glucose deprivation on selenium compounds was formdependent.

\section{The sensitization effect of glucose limitation on selenite is attributed to elevated ROS generation}

Generation of ROS, mainly superoxide, plays a critical role in selenite-mediated cytotoxic effect on cancer cells[24-26]. We hypothesized that the enhanced cytotoxic effect by combination of selenite and glucose deprivation might be associated with boosted ROS generation. The changes of selenite-induced ROS in response to different concentrations of glucose were measured by flow cytometry following staining with DHE or DCFH-DA. As shown in Fig. 2A, at concentration of $2.5 \mathrm{mM}$ glucose, selenite-induced ROS was significant higher than that found at concentrations of 10 and $25 \mathrm{mM}$ glucose, indicating

glucose deprivation promoted ROS generation in response to selenite. To assess the role of elevated ROS in the enhanced cytotoxicity by combining selenite with glucose deprivation, we evaluated effect of ROS scavenger MnTMPyP on the enhanced cytotoxicity. As shown in Fig. 2B, the cytotoxicity induced by combination of selenite and glucose deprivation was nearly abolished in the presence of MnTMPyP. In 
agreement with the cytotoxicity inhibition, ROS induction by either selenite alone or in combination with glucose deprivation was completely scavenged by the antioxidant (Fig. 2C). The results clearly suggested that the boosted ROS generation by combination of selenite and glucose deprivation contributed to the enhanced cytotoxicity.

\section{Expression of SLC7A11 is up-regulated by selenite, accompanied by cystine accumulation, cysteine reduction and NADPH depletion in the context of glucose deprivation}

As mentioned above, SLC7A11-mediated uptake of extracellular cystine plays important role in regulating cellular redox homeostasis. We next asked whether the elevated ROS generation by the combination of selenite and glucose deprivation was associated with disruption of SLC7A11-regulated redox balance. We first examined effect of selenite on SLC7A11 expression, and results showed that exposure to selenite led to a concentration-dependent increase of SLC7A11 expression in HCT116 colon cancer cells. The upregulation of SLC7A11 by selenite was detected at all three levels of glucose, but stronger increase of SLC7A11 by selenite was found at concentration of $2.5 \mathrm{mM}$ glucose (Fig. 3A). In agreement with the increased SLC7A11 expression, intracellular level of cystine was significantly elevated at all three concentrations of glucose in response to selenite exposure (Fig. 3B). Accordingly, intracellular level of cysteine was increased at concentrations of 25 and $10 \mathrm{mM}$ glucose. In contrast, a dramatic reduction of intracellular level of cysteine was induced by selenite in the context of glucose deprivation (Fig. 3C), indicating the conversion of cystine to cysteine was inhibited under such condition. The conversion of cystine to cysteine is a NADPH-dependent reaction, we therefore questioned whether shortage of NADPH contributed to the accumulation of cystine and reduction of cysteine. As shown in Fig. 3D, glucose deprivation alone did not cause NADPH depletion, but a significant increased NADP+/NADPH ratio was detected with selenite exposure in the context of glucose deprivation, suggesting involvement of NADPH depletion in this redox imbalance. Consistent with the reduction of cysteine, intracellular level of GSH, a cysteine-based antioxidant, was significantly reduced by selenite under condition of glucose deprivation (Fig. 3E). Together, these results indicated a well correlation between elevated SLC7A11 expression and redox collapse in response to selenite exposure in the context of glucose deprivation. In addition, the elevated expression of SLC7A11 was also observed in LLC and A549 lung cancer cells, HepG2 liver cancer cells and MDA-MB-231 breast cancer cells (Fig. 3F). However, the up-regulation of SLC7A11 by selenite was not found in HK-2 normal kidney cells, suggesting that up-regulation of SLC7A11 by selenite is a specific event for cancer cells, which was consistent with the selective enhancement of selenitemediated cytotoxicity of cancer cells. We also analyzed effect of MSeA on SLC7A11 expression, and results showed that MSeA failed to induce up-regulation of SLC7A11, which was in line with lack of sensitization effect of glucose deprivation on MSeA.

\section{SLC7A11-mediated cystine accumulation and NADPH depletion contribute to the elevated ROS generation and enhanced cytotoxicity induction by combination of selenite and glucose deprivation}

To critically determine the functional role of selenite-mediated up-regulation of SLC7A11, we evaluated effect of SLC7A11 inhibition by its chemical inhibitor or knocking-down of SLC7A11 on selenite/glucose 
deprivation-induced cytotoxicity. SAS, a known SLC7A11 inhibitor, was used to inactivate its function. Under such condition, the changes of cell viability were measured by crystal violet staining. As shown in Fig. 4A-E, SLC7A11 inhibition by its inhibitor led to nearly abolishment of the cell viability reduction by the combination. Accordingly, ROS generation and cystine accumulation were blocked, and the dysregulated $\mathrm{NADP}+$ /NADPH ratio and reduced GSH were recovered when SLC7A11 was inhibited by SAS. Similar results were also observed with another SLC7A11 inhibitor MSG (Fig. 4F-H). Furthermore, the functional role of SLC7A11 was validated by siRNA approach, and results showed that knocking-down of SLC7A11 mitigated the reduction of cell viability induced by selenite/glucose deprivation (Fig. 4I). In addition, we found that supplementation of NADPH or 2-deoxy-glucose (2-DG, activating a product of pentose phosphate pathway to generate NADPH from NADP+) offered a significant protection on selenite/glucose deprivation-induced cytotoxicity (Fig. 4J-K). Taken together, these results indicated that up-regulation of SLC7A11 by selenite played a pivotal role in the sensitization effect of glucose deprivation on seleniteinduced cytotoxicity of cancer cells, which is attributed to SLC7A11-mediated cystine accumulation, NADPH depletion, GSH reduction and ROS generation.

\section{Selenite/glucose deprivation-induced cytotoxicity is independent of ferroptosis}

Cysteine deprivation is one of common means to induce cell ferroptosis[27-29]. The above data showed that the cysteine level was dramatically reduced by selenite/glucose deprivation. We assumed that ferroptosis was involved in cytotoxicity induction by selenite/glucose deprivation. It has been shown that glutamine metabolism, known as glutaminolysis (conversion of glutamine to alpha-ketoglutarate), is required for cysteine deprivation-induced ferroptosis[30, 31]. We first tested the effects of glutaminolysis inhibition by glutamine deprivation from medium on the cytotoxicity induction by selenite/glucose deprivation. As expected, glutamine deprivation reversed the cytotoxicity of selenite/low-glucose combination and reduced ROS level (Fig. 5A-B). However, DFO or Fer-1, two ferroptosis inhibitors, failed to offer such protection (Fig. 5C-D). Furthermore, no significant increase of LPO or LIP level (two biomarkers for ferroptosis) was detected in response to selenite/glucose deprivation. These results suggested that ferroptosis was not involved in selenite/glucose deprivation-induced cytotoxic effect (Fig. 5E-F).

The above data demonstrated that glutamine was necessary for the enhanced cytotoxicity induced by selenite/glucose deprivation. Intracellular glutamate generated from glutamine metabolism, and glutamine deprivation is supposed to cause reduction of glutamate, which might reduce SLC7A11mediated uptake of cystine. We therefore speculated that the protection on the cytotoxicity offered by glutamine deprivation was attributed to its ability to inhibit cystine-glutamate exchange, which was similar to the condition of SLC7A11 inhibition by its inhibitor. To test this hypothesis, we measured the changes of intracellular cystine, GSH levels and NADP+/NADPH ratio in response to glutamine deprivation. Results showed that accumulated intracellular cystine and increased NADP+/NADPH ratio by combination of selenite and glucose deprivation were abolished, while GSH level was partially recovered under glutamine deprivation (Fig. 5G-I), which was consistent with the reduced ROS (Fig. 5B). These results provided a mechanistic explanation for the protection offered by glutamine deprivation. 


\section{Fasting improves therapeutic efficacy of selenite in vivo}

Having found the sensitization effect of glucose deprivation on selenite-induced cytotoxicity of cancer cells, we next asked whether the enhanced effect could be achieved in vivo. HCT116 and LLC xenograft models were employed to evaluate the anticancer effect of selenite/fasting described in Materials and Methods. As shown in Fig. 6A-B, intermittent fasting caused a significant inhibition of tumor growth and reduction of tumor weight, whereas selenite alone did not. Selenite/fasting combination further significantly delayed the tumor growth and reduced the tumor weight. Consistent with the in vitro findings, selenite/fasting combination decreased GSH levels and increased NADP+/NADPH ratio, which reflected the redox imbalance in selenite/fasting combination-treated tumors (Fig. 6C-D). Moreover, SLC7A11 expression was significantly up-regulated in response to treatment with selenite alone or in combination with fasting (Fig. 6E). The combination did not cause decrease of bodyweight in comparison with fasting alone, indicating no increased toxicity by the combination (data not shown). These results suggested that the fasting was able to improve efficacy of selenite against colon and lung cancer in xenograft models, which was associated with up-regulation of SLC7A11 expression and induction of oxidative stress.

\section{Discussion}

Dose-limiting toxicity of selenite is still a major concern for its clinical use even though the selenophilic feature of cancer cells. Approaches that can potentiate cancer cells to selenite are clearly needed for promoting selenite as a clinical useful anticancer agent. In the present study, we demonstrated that the oxidative stress and cytotoxic effect induced by selenite were amplified in the context of glucose limitation, mechanistically associated with SLC7A11-mediated cystine accumulation, NADPH depletion, inhibition of cystine to cysteine conversion, and redox collapse. Moreover, this sensitization effect on cancer cells was not observed on the normal cells. Accordingly, the in vivo efficacy was significantly improved by the combination of selenite and fasting treatment without increased toxicity. The findings of the present study provide an effective and practical approach for increasing the therapeutic window of selenite and promoting the development of selenite as a clinically useful selective anticancer agent.

SLC7A11-mediated uptake of cysine plays an important role in maintaining redox homeostasis[32]. Conversion of cystine to cysteine is a critical step for cystine-mediated antioxidant function[33]. NADPH, a product of pentose phosphate pathway, is required for the conversion of cystine to cysteine to synthesize intracellular antioxidant glutathione (GSH)[34, 35]. In the present study, we found that SLC7A11 expression was up-regulated by selenite in all cancer cell lines tested either in glucose replete or starvation condition. Up-regulation of SLC7A11 is supposed to promote cystine uptake, which is rapidly converted to cysteine, leading to elevated GSH levels in the context of glucose replete. Indeed, our data showed that selenite-induced SLC7A11 resulted in increase of cysteine and GSH under glucose sufficient condition. This SLC7A11-mediated increase of antioxidant capacity might compromise the oxidative stress and cytotoxic effect induced by selenite in the context of sufficient glucose. In contrast, under the condition of glucose limitation, the up-regulation of SLC7A11 by selenite was accompanied by increased 
cystine accumulation, decreased cysteine and GSH, followed by elevated ROS generation and cell death induction. In other words, glucose deprivation disrupted SLC7A11-cystine-cysteine-GSH-mediated antioxidant system, which in turn boosted selenite-induced oxidative stress and cytotoxic effect against cancer. NADPH availability is supposed to be responsible for this paradoxical role of SLC7A11 in regulating redox balance in different condition of glucose. Glucose is a major source for generating NADPH, high NADPH consumption due to SLC7A11-mediated increase of cystine uptake may rapidly cause NADPH depletion under glucose deprivation, which in turn led to disruption of cystine-cysteine-GSH metabolic axis. It was worth to point out that the sensitization effect of glucose limitation on cancer cells in response to selenite was not observed in normal lung cells, which was consistent with that the upregulation of SLC7A11 by selenite in cancer cells but not found in normal cells, further supporting the role of SLC7A11 in glucose starvation-mediated sensitization effect on cancer cells. The mechanisms underlying these differential effect of selenite on SLC7A11 expression between cancer cells and normal cells need to be further investigated.

Oxidative stress induction by selenite is well established, which is suggested to play a pivotal role in its cytotoxic effect on cancer cells. It has been shown that oxidative stress induction can sensitize cancer cells to a variety of anticancer agents and radiotherapy[8,36,37]. Our previous study has shown that selenite sensitized LNCaP prostate cancer cells to TRIAL-induced apoptosis, which is due to its ability to generate ROS[38]. A sensitization effect of selenite on refractory prostate cancer cells to radiation is also associated with the redox status[19]. The induction of oxidative stress by selenite was boosted in the context of glucose starvation, we therefore speculated that combination of selenite with glucose deprivation could produce a stronger sensitization effect on cancer cells in response to certain types of therapeutic treatments. This hypothesis needs to investigate in the follow-up studies.

As mentioned above, the form of selenium is an important factor affecting its anticancer activity. In the preventive setting, Larry Clark et al[22] demonstrated that supplementation of selenium (Se) in the form of selenized yeast, which contains multiple forms of selenium compounds, leads to reduction of cancer risk, especially the cancer of prostate, lung and colon. However, a large-scale human intervention with selenomethionine (SeMet) supplementation (the selenium and vitamin E cancer prevention trial, SELECT) in North America failed to achieve an inhibitory effect on prostate carcinogenesis[39]. One possible reason for these controversial outcomes is an incorrect choice of selenium form for this clinical study in a context of selenium- adequate condition. This notion is supported by our previous study, in which, MSeA, but not selenite or SeMet is effective in a xenograft model of prostate cancer[40]. In the present experimental setting, our data showed that selenite alone was still ineffective, but combining glucose limitation with selenite produced a significantly enhanced anticancer effect in the two xenograft models. The sensitization effect was not induced by combining glucose limitation with MSeA. These data support a context-dependent nature of selenium compound-mediated anticancer effect. The sensitization effect of glucose limitation on selenite requires SLC7A11-meidated redox collapse. This event was not observed in response to MSeA, providing possible interpretation for the lack of sensitization effect of glucose limitation on MSeA. 


\section{Conclusion}

In summary, SLC7A11 was up-regulated by selenite exposure both in vitro and in vivo. The elevated expression of SLC7A11 by selenite resulted in cystine accumulation, NADPH depletion and redox collapse in the context of glucose starvation. Under such condition, the cytoctoxic effect of selenite on cancer cells was specifically enhanced. Moreover, the therapeutic efficacy of selenite in vivo was greatly improved in xenograft models of lung and colon cancer when the treatment was coupled with fasting. The findings of the present study suggest that combination of selenite with fasting holds promising potential to be developed as an effective treatment regimen for certain types of cancer.

\section{Abbreviations}

SLC7A11: solute carrier family 7 member 11, ROS: reactive oxygen species, DHE: dihydroethidium, DCFHDA: 2',7'- dichlorodihydrofluorescein diacetate, MnTMPyP: manganese (III) tetrakis (1-methyl-4-pyridyl) porphyrin, Cyss: cystine, Cys: cysteine, NADP+: oxidized form of nicotinamide-adenine dinucleotide phosphate, NADPH: reduced form of nicotinamide-adenine dinucleotide phosphate, GSH: glutathione, GIn: glutamine, DFO: deferoxamine mesylate, Fer-1: ferrostatin-1, MSG: monosodium glutamate, SAS: salicylazosulfapyridine, 2-DG: 2-deoxy-D-glcose, LC-MS: liquid chromatograph-mass spectrometer, LIP: labile iron pool, LPO: lipid peroxidation, T-BHP: tert-butyl hydroperoxide, FAS: ferrous ammonium sulfate.

\section{Declarations}

\section{Author contributions}

$\mathrm{HBH}$ and $\mathrm{HC}$ designed the study, $\mathrm{HC}, \mathrm{HZ}$ and $\mathrm{LXC}$ carried out the experiments, and conducted data collection. $\mathrm{HBH}, \mathrm{HC}, \mathrm{HZ}, \mathrm{LXC}, \mathrm{JLC}, \mathrm{XM}, \mathrm{CZ}$ and STY analyzed the data and discussion. $\mathrm{HBH}$ and $\mathrm{HC}$ wrote the manuscript. All authors read and approved the final manuscript.

\section{Funding}

This study was supported by the Ministry of Science and Technology of China, National Key Research and Development Program of China (Grant No. 2018YFC1603706).

\section{Availability of data and materials}

Hongbo Hu and Hui Chen had the full access to all the data in the study and had final responsibility for the decision to submit for publication. And we declared that materials described in the manuscript, including relevant data, will be freely available to any scientist wishing to use them after informing Hongbo Hu and Hui Chen.

\section{Ethics approval and consent to participate}


This study was approved by the Ethics Committee of Shenzhen Peking University-The Hong Kong University of Science and Technology Medical Center (No.2020-328).

Principles of China Agricultural University Institutional Animal Care and Use Committee (No.2021-0045-2)

\section{Consent for publication}

This manuscript has been viewed and approved by all authors for publication.

\section{Conflict of interest}

The authors declare that they have no conflict of interest.

\section{References}

1. M. Elgendy, M. Cirò, A. Hosseini, J. Weiszmann, L. Mazzarella, E. Ferrari, et al., Combination of hypoglycemia and metformin impairs tumor metabolic plasticity and growth by modulating the PP2A-GSK3B-MCL-1 axis, Cancer Cell 35 (2019) 798-815.

2. S.W. Kim, M. Cha, S. Lee, B. Song, X. Jin, J.M. Lee, et al., Curcumin treatment in combination with glucose restriction inhibits intracellular alkalinization and tumor growth in hepatoma cells, Int J Mol Sci 20 (2019) 2375-2391.

3. M. Di Tano, F. Raucci, C. Vernieri, I. Caffa, R. Buono, M. Fanti, et al., Synergistic effect of fastingmimicking diet and vitamin C against KRAS mutated cancers, Nat Commun 11 (2020) 2332-2342.

4. X. Tang, G. Li, L. Shi, F. Su, M. Qian, Z. Liu, et al., Combined intermittent fasting and ERK inhibition enhance the anti-tumor effects of chemotherapy via the GSK3beta-SIRT7 axis, Nat Commun 12 (2021) 5058-5075.

5. B. Bhattacharya, M.F. Mohd Omar, R. Soong, The Warburg effect and drug resistance, Brit J Pharmacol 173 (2016) 970-979.

6. K. Birsoy, R. Possemato, F.K. Lorbeer, E.C. Bayraktar, P. Thiru, B. Yucel, Tet al., Metabolic determinants of cancer cell sensitivity to glucose limitation and biguanides, Nature (London) 508 (2014) 108-112.

7. G. Bianchi, R. Martella, S. Ravera, A. Petretto, D. Marimpietri, L. Emionite, et al., Fasting chemosensitizes tumor cells by affecting their metabolism, Cancer Res 74 (2014) 3374-3374.

8. C. Mølck, J. Ryall, L.M. Failla, J.L. Coates, J. Pascussi, J.K. Heath, et al., The A2b adenosine receptor antagonist PSB-603 promotes oxidative phosphorylation and ROS production in colorectal cancer cells via adenosine receptor independent mechanism, Cancer Lett 383 (2016) 135-143.

9. X. Liu, K. Olszewski, Y. Zhang, E.W. Lim, J. Shi, X. Zhang, et ai., Cystine transporter regulation of pentose phosphate pathway dependency and disulfide stress exposes a targetable metabolic vulnerability in cancer, Nat Cell Biol 22 (2020) 476-486.

10. E. Olm, A.P. Fernandes, C. Hebert, A. Rundlöf, E.H. Larsen, O. Danielsson, et al., Extracellular thiolassisted selenium uptake dependent on the $\mathrm{xc}^{-}$cystine transporter explains the cancer-specific 
cytotoxicity of selenite, PNAS 106 (2009) 11400-11405.

11. A.E. Carlisle, N. Lee, A.N. Matthew-Onabanjo, M.E. Spears, S.J. Park, D. Youkana, et al., Selenium detoxification is required for cancer-cell survival, Nat Metab 2 (2020) 603-611.

12. P. Koppula, L. Zhuang, B. Gan, Cystine transporter SLC7A11/xCT in cancer: ferroptosis, nutrient dependency, and cancer therapy, Protein Cell 12 (2021) 599-620.

13. S. Bekeschus, S. Eisenmann, S.K. Sagwal, Y. Bodnar, J. Moritz, B. Poschkamp, et al., xCT (SLC7A11) expression confers intrinsic resistance to physical plasma treatment in tumor cells, Redox Biol 30 (2020) 101423-101432.

14. W. Hu, C. Zhao, H. Hu, S. Yin, Food sources of selenium and its relationship with chronic diseases, Nutrients 13 (2021) 1739-1761.

15. R.R. Cavalieri, K.G. Scott, E. Sairenji, Selenite (75Se) as a tumor-localizing agent in man, J Nucl Med 7 (1966) 197-208.

16. X. Wu, G. Zhao, Y. He, W. Wang, C.S. Yang, J. Zhang, Pharmacological mechanisms of the anticancer action of sodium selenite against peritoneal cancer in mice, Pharmacol Res 147 (2019) 104360104369.

17. W. Chen, J. An, J. Guo, Y. Wu, L. Yang, J. Dai, et al., Sodium selenite attenuates lung adenocarcinoma progression by repressing SOX2-mediated stemness, Cancer Chemother Pharmacol 81 (2018) 885895.

18. T. Zhang, G. Zhao, X. Zhu, K. Jiang, H. Wu, G. Deng, et al., Sodium selenite induces apoptosis via ROS-mediated NF-KB signaling and activation of the Bax caspase-9-caspase-3 axis in 4T1 cells, $\mathrm{J}$ Cell Physiol 234 (2019) 2511-2522.

19. B. Husbeck, D.M. Peehl, S.J. Knox, Redox modulation of human prostate carcinoma cells by selenite increases radiation-induced cell killing, Free Radic Biol Med 38 (2005) 50-57.

20. O. Brodin, S. Eksborg, M. Wallenberg, C. Asker-Hagelberg, E.H. Larsen, D. Mohlkert, et al., Pharmacokinetics and toxicity of sodium selenite in the treatment of patients with carcinoma in a phase I clinical trial: the SECAR study, Nutrients 7 (2015) 4978-4994.

21. G.D. Frenkel, P.B. Caffrey, Prevention of carboplatin-induced resistance in human ovarian tumor xenografts by selenite, Anticancer Res 33 (2013) 4249-4254.

22. R. Zhao, N. Xiang, F.E. Domann, W. Zhong, Expression of p53 enhances selenite-induced superoxide production and apoptosis in human prostate cancer cells, Cancer Res 66 (2006) 2296-2304.

23. M. Yan, L. Ye, S. Yin, X. Lu, X. Liu, S. Lu, et al., Glycycoumarin protects mice against acetaminopheninduced liver injury predominantly via activating sustained autophagy, Brit J Pharmacol 175 (2018) 3747-3757.

24. H. Wang, X. Yang, Z. Zhang, H. Xu, Both calcium and ROS as common signals mediate NaSeO3induced apoptosis in SW480 human colonic carcinoma cells, J Inorg Biochem 97 (2003) 221-230.

25. E.H. Kim, S. Sohn, H.J. Kwon, S.U. Kim, M. Kim, S. Lee, et al., Sodium selenite induces superoxidemediated mitochondrial damage and subsequent, Cancer Res 67 (2007) 6314-6324. 
26. W. Zhong, T.D. Oberley, Redox-mediated effects of selenium on apoptosis and cell cycle in the LNCaP human prostate cancer cell line, Cancer Res 61 (2001) 7071-7078.

27. M. Gao, J. Yi, J. Zhu, A.M. Minikes, P. Monian, C.B. Thompson, et al., Role of mitochondria in ferroptosis, Mol Cell 73 (2019) 354-363.

28. P. Liu, D. Wu, J. Duan, H. Xiao, Y. Zhou, L. Zhao, et al., NRF2 regulates the sensitivity of human NSCLC cells to cystine deprivation-induced ferroptosis via FOCAD-FAK signaling pathway, Reddox Biol 37 (2020) 101702-101714.

29. Y.P. Kang, A. Mockabee-Macias, C. Jiang, A. Falzone, N. Prieto-Farigua, E. Stone, et al., Non-canonical Glutamate-Cysteine Ligase Activity Protects against Ferroptosis, Cell Metab 33 (2021) 174-189.

30. M. Gao, P. Monian, X. Jiang, Metabolism and iron signaling in ferroptotic cell death, Oncotarget 6 (2015) 35145-35146.

31. M. Gao, P. Monian, N. Quadri, R. Ramasamy, X. Jiang, Glutaminolysis and transferrin regulate ferroptosis, Mol Cell 59 (2015) 298-308.

32. M. Lo, Y.Z. Wang, P.W. Gout, The $x(c)$ - cystine/glutamate antiporter: a potential target for therapy of cancer and other diseases, J Cell Physiol 215 (2008) 593-602.

33. P. Koppula, Y. Zhang, L. Zhuang, B. Gan, Amino acid transporter SLC7A11/xCT at the crossroads of regulating redox homeostasis and nutrient dependency of cancer, Cancer Commun 38 (2018) 1-13.

34. X. Liu, Y. Zhang, L. Zhuang, K. Olszewski, B. Gan, NADPH debt drives redox bankruptcy: SLC7A11/xCT-mediated cystine uptake as a double-edge sword in cellular redox regulation. Genes \& diseases 6 (2021) 731-745.

35. M. Deponte, Glutathione catalysis and the reaction mechanisms of glutathione-dependent enzymes, BBA-Gen Subjects 1830 (2013) 3217-3266.

36. Q. Wu, A. Allouch, A. Paoletti, C. Leteur, C. Mirjolet, I. Martins, et al., NOX2-dependent ATM kinase activation dictates pro-inflammatory macrophage phenotype and improves effectiveness to radiation therapy, Cell Death Differ 24 (2017) 1632-1644.

37. H.J. Kim, K.H. Min, H.J. Lee, Y. Hwang, S.C. Lee, Fenton-like reaction performing mineralized nanocarriers as oxidative stress amplifying anticancer agents, J Ind Eng Chem 80 (2019) 829-837.

38. H. Hu, C. Jiang, T. Schuster, G. Li, P.T. Daniel, J. Lu, Inorganic selenium sensitizes prostate cancer cells to TRAIL-induced apoptosis through superoxide/p53/Bax-mediated activation of mitochondrial pathway, Mol Cancer Ther 5 (2006) 1873-1882.

39. E.A.A. Klein, I.M.B. Thompson Jr., C.M.C. Tangen, J.J.C. Crowley, S.D. Lucia, P.J.C. Goodman, et al., Vitamin $E$ and the risk of prostate cancer: The selenium and vitamin E cancer prevention trial (SELECT)(Article), JAMA-J Am Med Assoc 306 (2011) 1549-1556.

40. G. Li, H. Lee, Z. Wang, H. Hu, J.D. Liao, J.C. Watts, et al., Superior in vivo inhibitory efficacy of methylseleninic acid against human prostate cancer over selenomethionine or selenite, Carcinogenesis (New York) 29 (2008) 1005-1012. 
D $25 \mathrm{mM}$ glucose $10 \mathrm{mM}$ glucose $\quad 2.5 \mathrm{mM}$ glucose

A

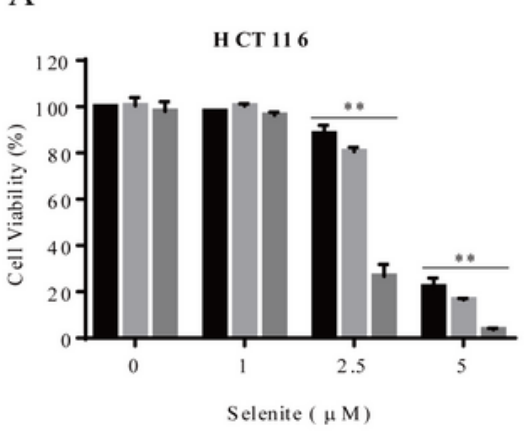

D

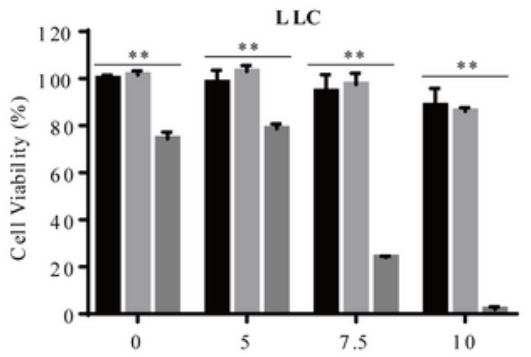

Selenite $(\mu \mathrm{M})$
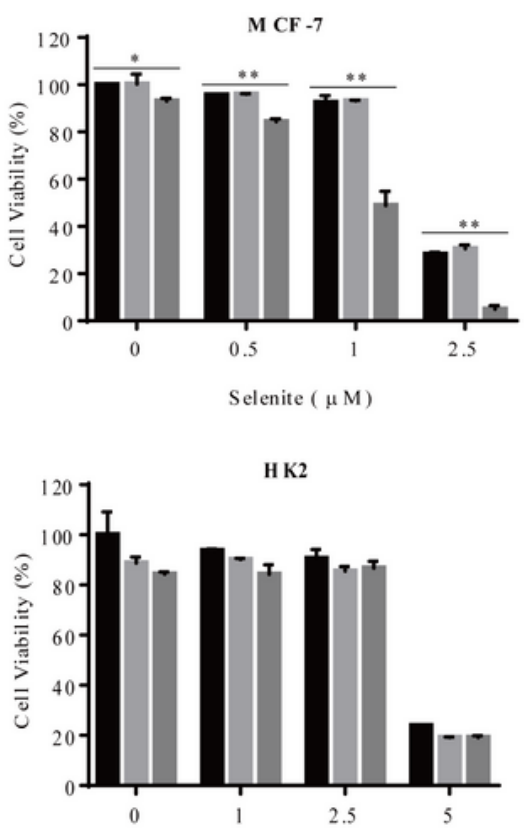

Selenite $(\mu \mathrm{M})$
B

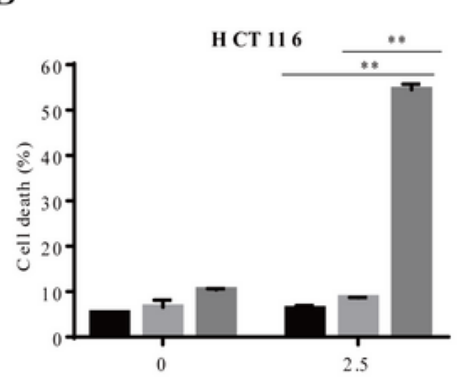

Selenite ( $\mu \mathrm{M})$
C

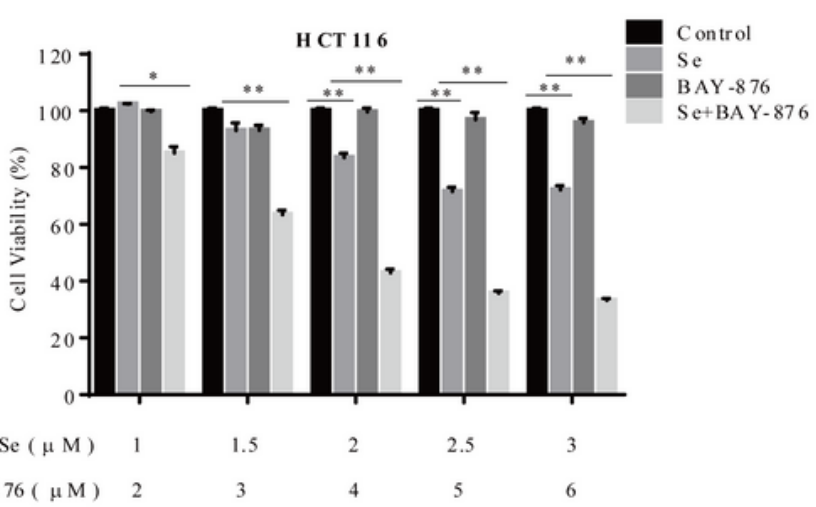

$25 \mathrm{mM}$ glucose $\quad 10 \mathrm{mM}$ glucose $\quad 2.5 \mathrm{mM}$ glucose

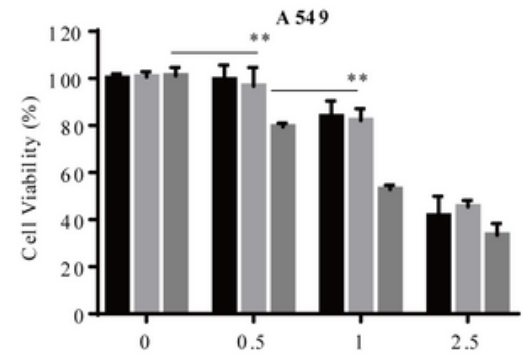

Selenite $(\mu \mathrm{M})$
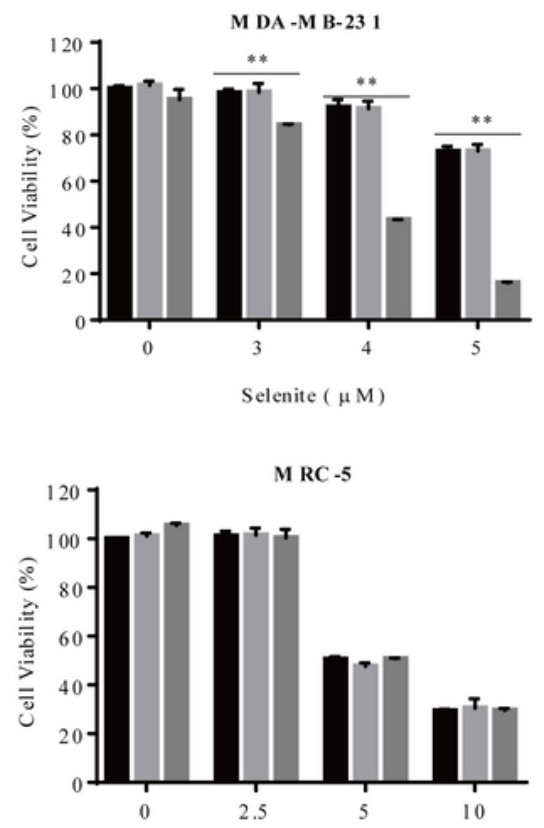

Selenite $(\mu \mathrm{M})$

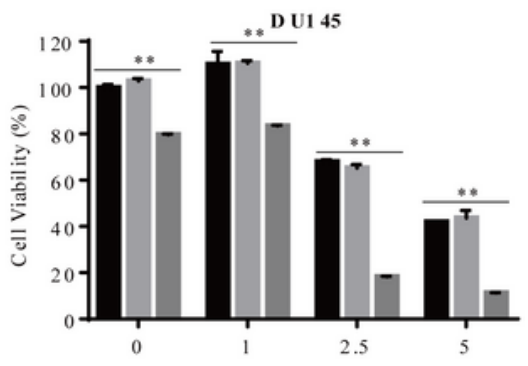

Selenite ( $\mu \mathrm{M})$

E

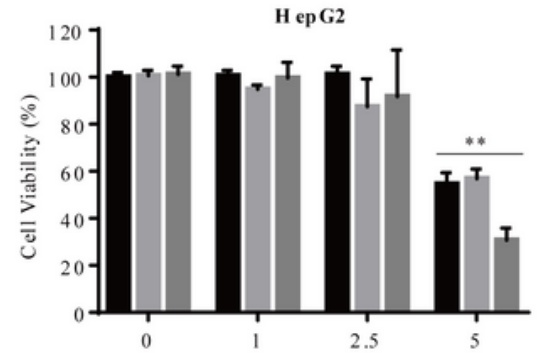

Selenite $(\mu \mathrm{M})$

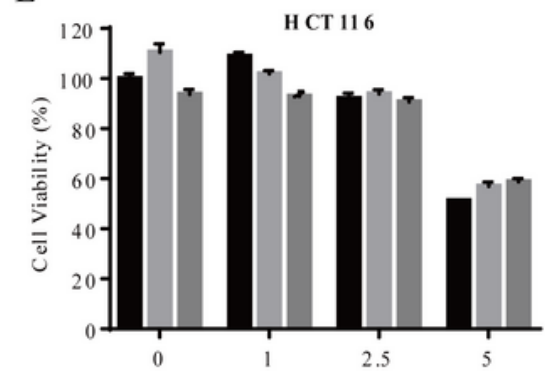

$\mathrm{MSeA}(\mu \mathrm{M})$

Figure 1

Glucose limitation sensitizes cancer cells to selenite-mediated cytotoxic effect. A-B Cell viability (A) and cell death (B) of HCT116 cells cultured in the medium containing indicated concentrations of glucose with or without treatment of selenite for $24 \mathrm{~h}$. C Cell viability of HCT116 cells with or without treatment of 
selenite, BAY-876 or selenite/BAY-876 combination for 24 h. D Cell viability of LLC, A549, HepG2, MCF-7, MDA-MB-231, DU145, HK2 and MRC-5 cells cultured in the medium containing indicated concentrations of glucose with or without treatment of selenite for $24 \mathrm{~h}, 36 \mathrm{~h} \varangle 36 \mathrm{~h}, 48 \mathrm{~h}, 36 \mathrm{~h}, 48 \mathrm{~h}, 48 \mathrm{~h}$ and $48 \mathrm{~h}$, respectively. E Cell viability of HCT116 cells cultured in the medium containing indicated concentrations of glucose with or without treatment of methylseleninic acid for $24 \mathrm{~h}$. Results are representative of three biologically independent experiments. Data are expressed as mean $\pm S D$, ${ }^{\star} p<0.05,{ }^{*} p<0.01$. Se: selenite, MSeA: methylseleninic acid.

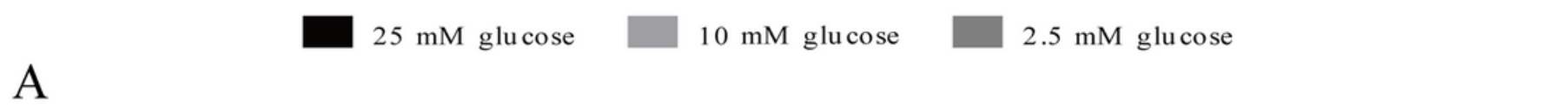

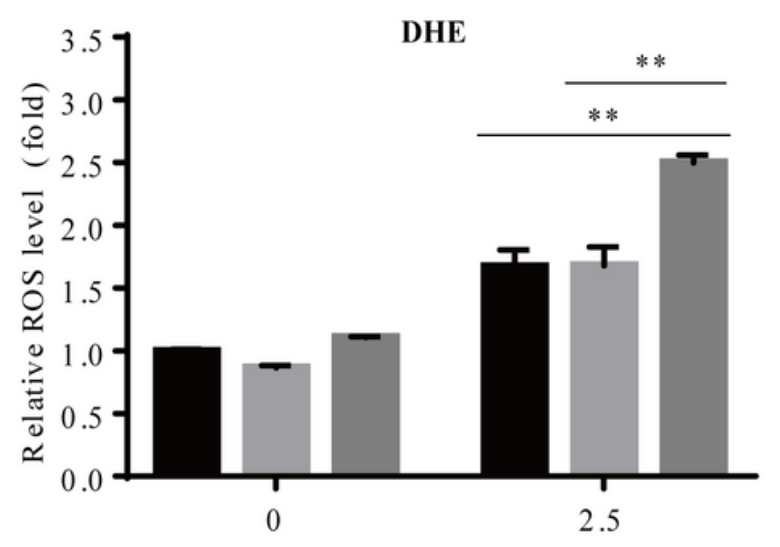

$\mathrm{B}$

Selenite $(\mu \mathrm{M})$

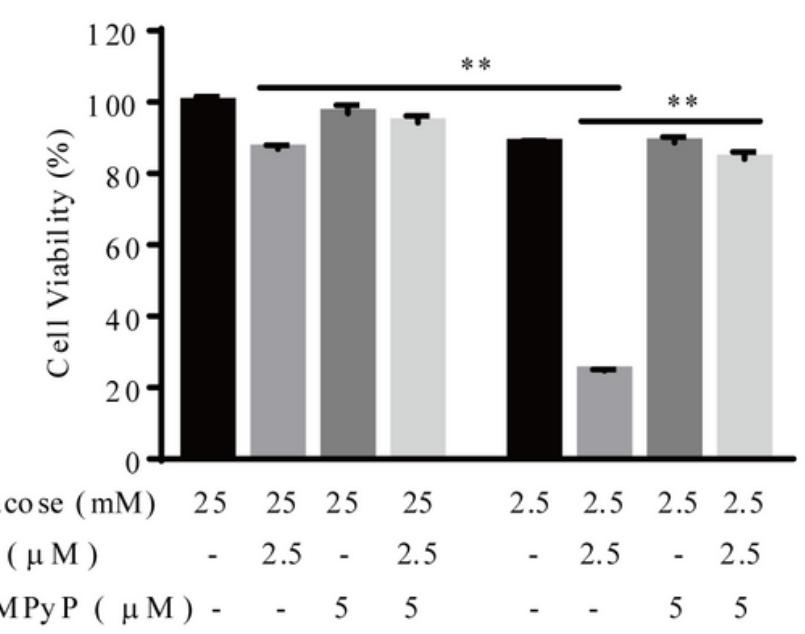

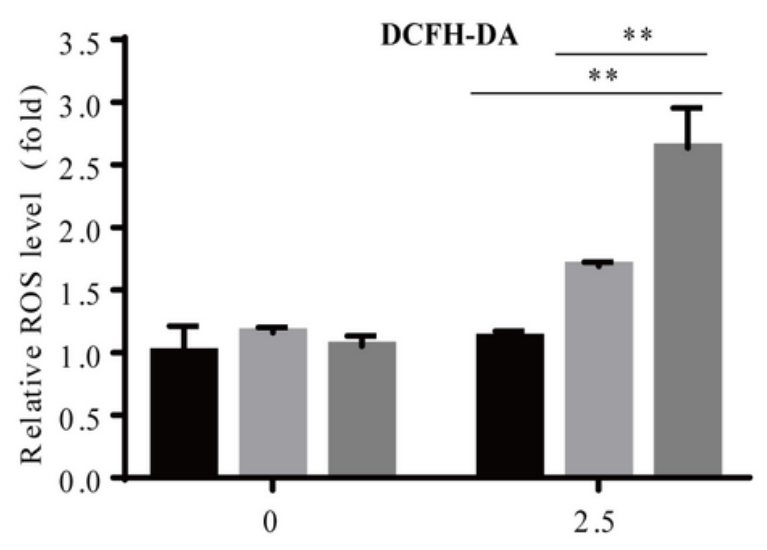

Selenite $(\mu \mathrm{M})$

$\mathrm{C}$

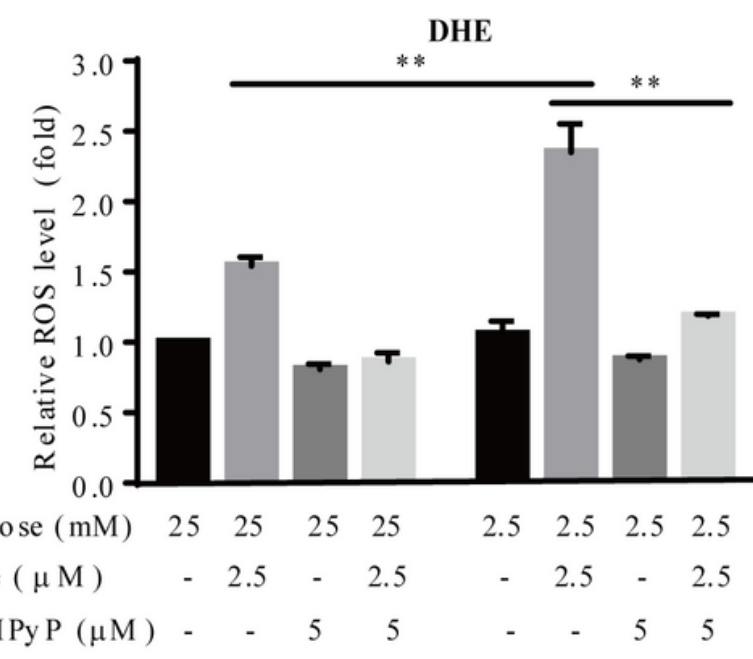

\section{Figure 2}

The sensitization effect of glucose limitation on selenite is attributed to elevated ROS generation. A The intracellular ROS level staining with DHE ( for 02--) or DCFH-DA (for H2O2) of HCT116 cells cultured in the medium containing indicated concentrations of glucose with or without treatment of selenite for $22 \mathrm{~h}$. B Cell viability of HCT116 cells cultured in the medium containing 25 or $2.5 \mathrm{mM}$ glucose with or without treatment of selenite or MnTMPyP for $24 \mathrm{~h}$. C 02•- levels of HCT116 cells cultured in the medium 
containing 25 or $2.5 \mathrm{mM}$ glucose with or without treatment of selenite or MnTMPyP $22 \mathrm{~h}$. Results are representative of three biologically independent experiments. Data are expressed as mean $\pm S D, * \star p<0.01$. MnTMPyP: manganese (III) tetrakis (1-methyl-4-pyridyl) porphyrin.

A

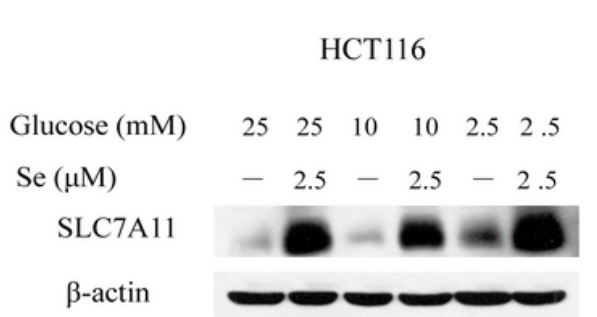

$\mathrm{D}$

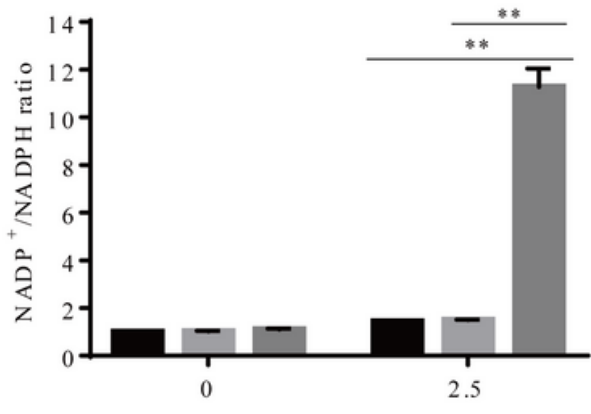

Selenite $(\mu \mathrm{M})$
$\mathrm{B}$

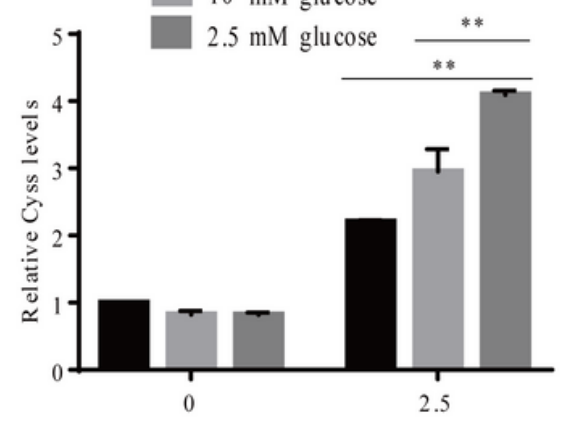

Selenite $(\mu \mathrm{M})$
$\mathrm{C}$

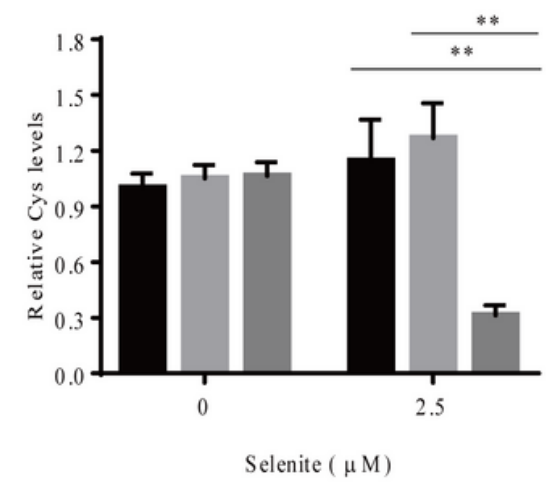

$\mathrm{E}$

$\mathrm{F}$

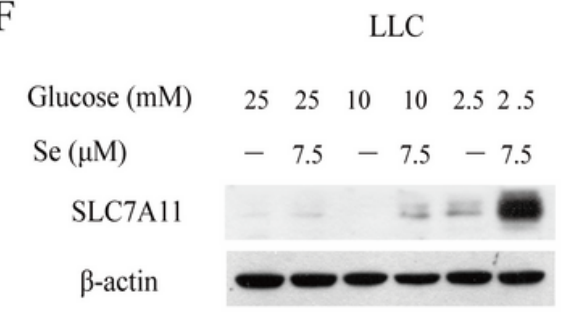

A549

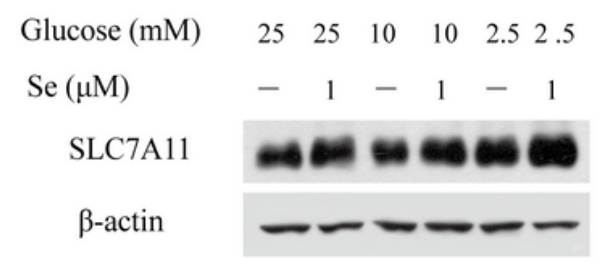

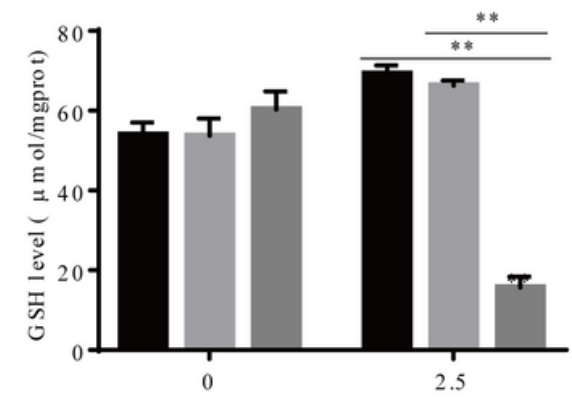

Selenite $(\mu \mathrm{M})$

HepG2

HK2

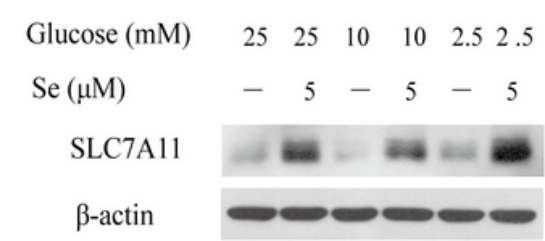

MDA-MB-231

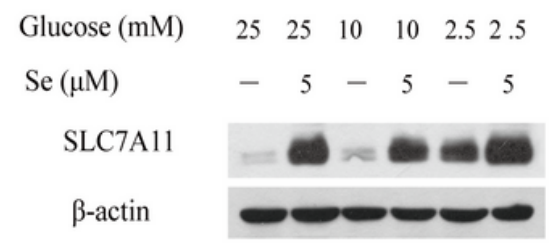

HCT116

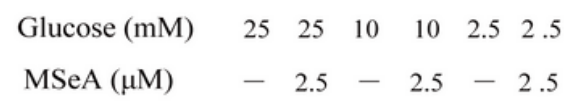

SLC7A11

$\beta$-actin

\section{Figure 3}

Expression of SLC7A11 is up-regulated by selenite, accompanied by cystine accumulation, cysteine reduction and NADPH depletion in the context of glucose deprivation. A SLC7A11 protein levels of HCT116 treated with or without selenite in different concentrations of medium glucose for $22 \mathrm{~h}$. B-E Cyss levels (B), Cys levels (C), NADP+/NADPH ratios (D) and GSH levels (E) of HCT116 cells cultured in the 
medium containing indicated concentrations of glucose with or without treatment of selenite for $22 \mathrm{~h}$. $F$ SLC7A11 protein levels of LLC, A549, MDA-MB-231, HepG2 and HK2 cells treated with or without selenite in different concentnations of medium glucose for $22 \mathrm{~h}, 30 \mathrm{~h}, 30 \mathrm{~h}, 30 \mathrm{~h}$ and $48 \mathrm{~h}$, respectively, SLC7A11 protein levels of HCT116 cells treated with or without MSeA in different concentnations of medium glucose for $22 \mathrm{~h}$. Results are representative of three biologically independent experiments. Data are expressed as mean $\pm S D$, ${ }^{\star *} p<0.01$. Cyss: cystine, Cys: cysteine, NADP+: oxidized form of nicotinamideadenine dinucleotide phosphate, NADPH: reduced form of nicotinamide-adenine dinucleotide phosphate, GSH: glutathione, Se: selenite, MSeA: methylseleninic acid.

A

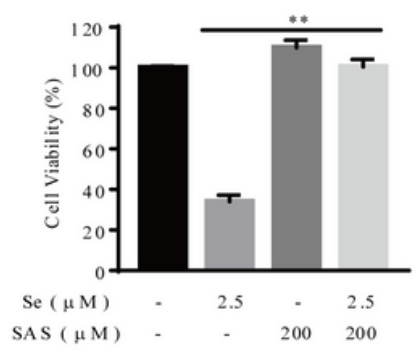

$\mathrm{E}$

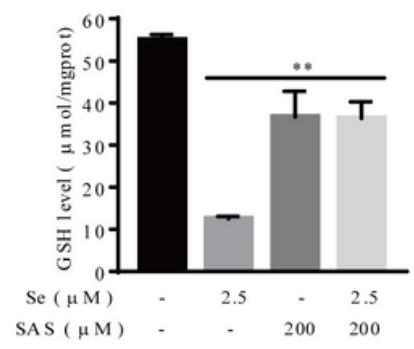

I

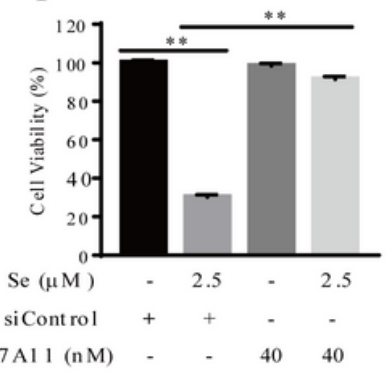

B

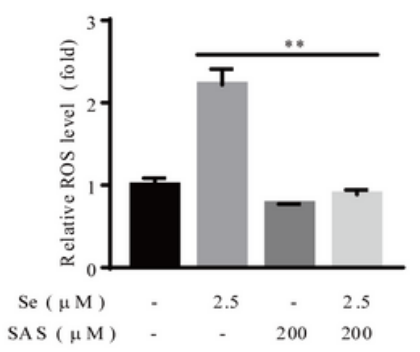

F

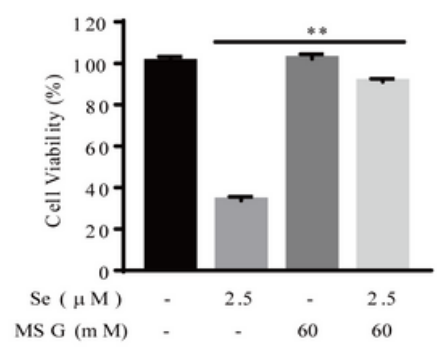

C

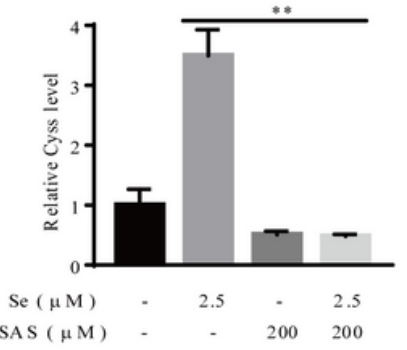

G

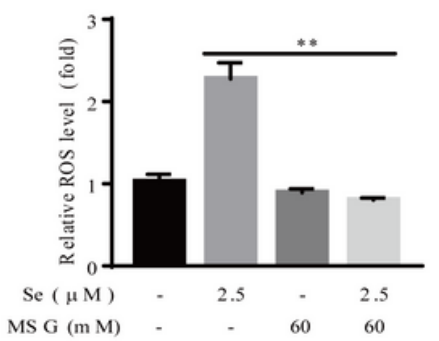

J

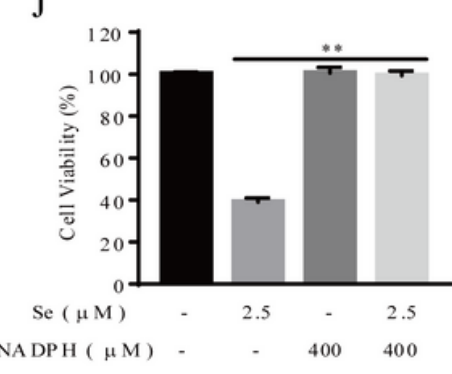

D

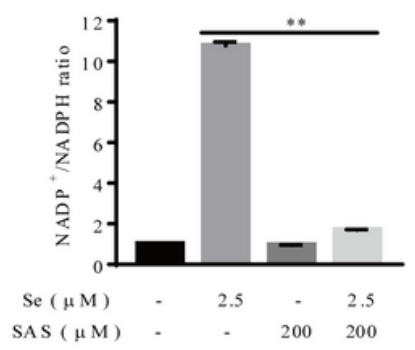

H

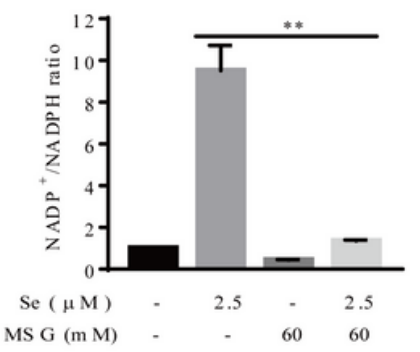

K

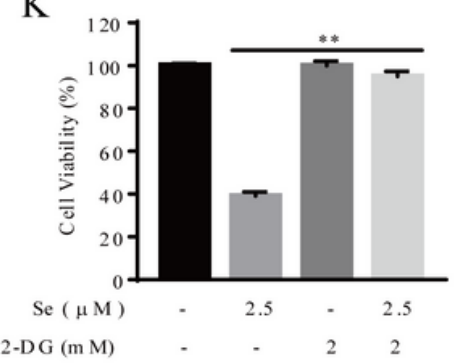

\section{Figure 4}

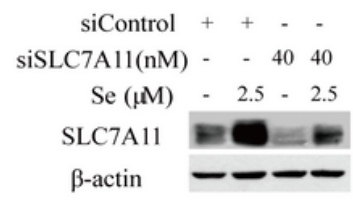

SLC7A11-mediated cystine accumulation and NADPH depletion contributes to the elevated ROS generation and enhanced cytotoxicity induction by combination of selenite and glucose deprivation. A-E Cell viability (A), 02•- levels (B), Cys levels (C), NADP+/NADPH ratios (D) and GSH levels (E) of HCT116 cells cultured in the medium containing $2.5 \mathrm{mM}$ glucose with or without treatment of selenite or MSG for $24 \mathrm{~h}(\mathrm{~A})$ and $22 \mathrm{~h}(\mathrm{~B}-\mathrm{E})$. F-H Cell viability (F), 02-- levels (G) and NADP+/NADPH ratios (H) of HCT116 cells cultured in the medium containing $2.5 \mathrm{mM}$ glucose with or without treatment of selenite or SAS for $24 \mathrm{~h}(\mathrm{~F})$ and $22 \mathrm{~h}(\mathrm{G}, \mathrm{H})$. I Cell viability and SLC7A11 protein levels of HCT116 cells expressing either 
scrambled siRNA or siRNA against SLC7A11 cultured in the medium containing $2.5 \mathrm{mM}$ glucose with or without treatment of selenite for $24 \mathrm{~h}$ and $22 \mathrm{~h}$, respectively. J-K Cell viability of HCT116 cells cultured in the medium containing $2.5 \mathrm{mM}$ glucose with or without treatment of selenite or NADPH or 2-DG for $24 \mathrm{~h}$. Results are representative of three biologically independent experiments. Data are expressed as mean $\pm S D$, $* \star p<0.01$. Se: selenite, SAS: salicylazosulfapyridine, Cyss: cystine, NADP+: oxidized form of nicotinamide-adenine dinucleotide phosphate, NADPH: reduced form of nicotinamide-adenine dinucleotide phosphate, GSH: glutathione, MSG: monosodium glutamate, 2-DG: 2-deoxy-D-glucose.

A

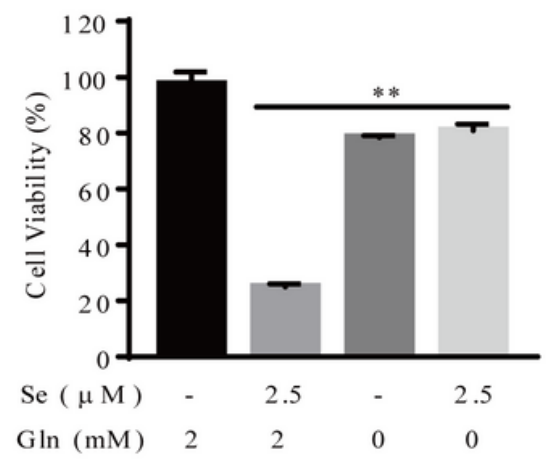

$\mathrm{D}$

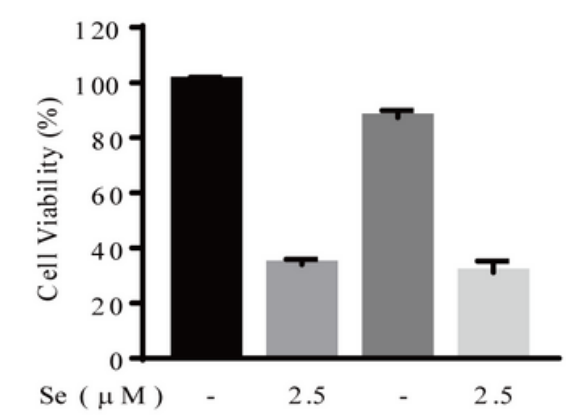

Fer-1 ( $\mu \mathrm{M})$

$\mathrm{G}$

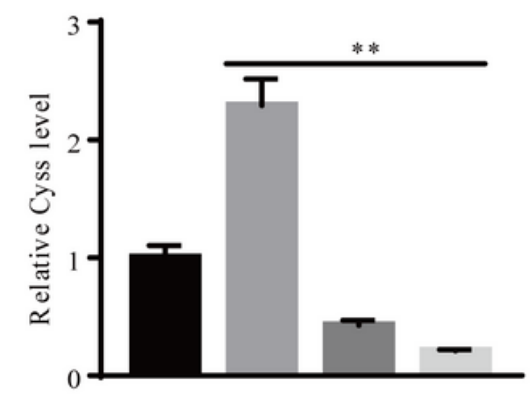

Se $(\mu \mathrm{M}) \quad-\quad 2.5 \quad-\quad 2.5$

$\mathrm{G} \ln (\mathrm{mM}) \quad 2 \quad 2 \quad 000$
$\mathrm{B}$

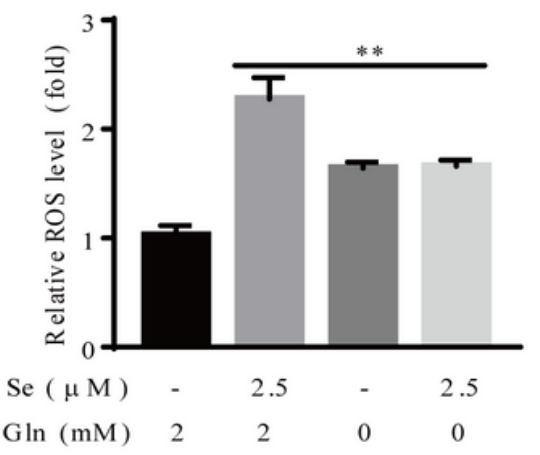

$\mathrm{E}$

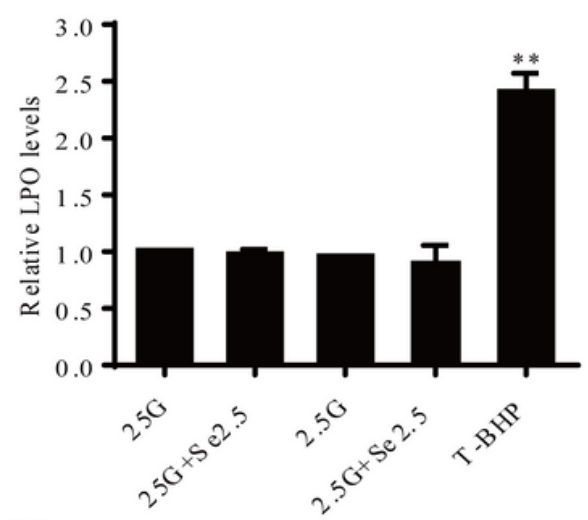

$\mathrm{H}$

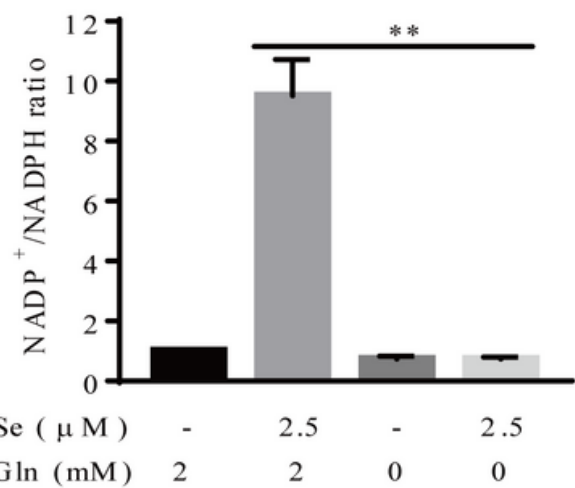

$\mathrm{C}$

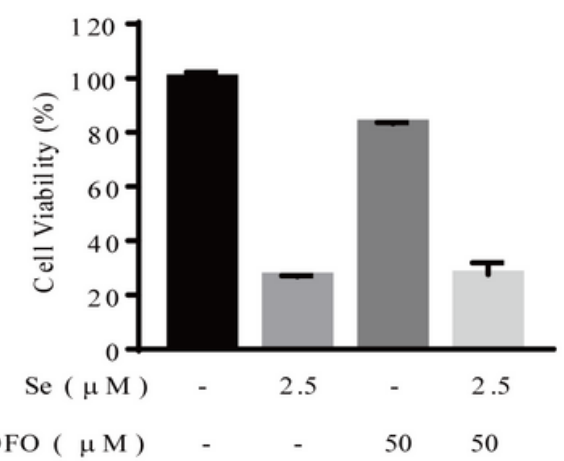

$\mathrm{F}$

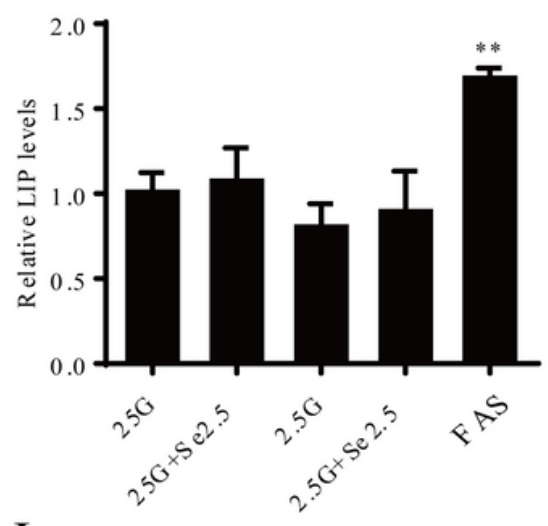

I

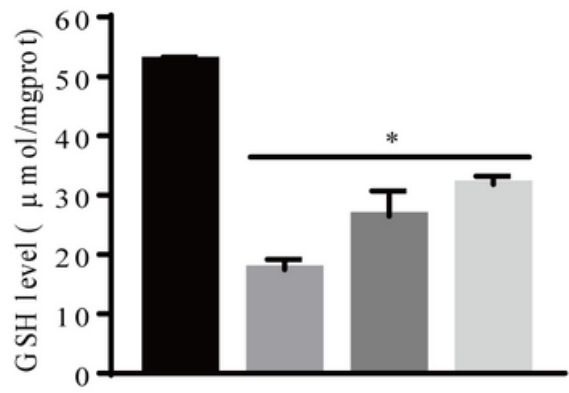

$\operatorname{Se}(\mu \mathrm{M}) \quad-\quad 2.5 \quad-\quad 2.5$ $\mathrm{G} \ln (\mathrm{mM}) \quad 2 \quad 2 \quad 000$

\section{Figure 5}

Selenite/glucose deprivation-induced cytotoxicity is independent of ferroptosis. A-B Cell viability (A) and 02•- levels (B) of HCT116 cells cultured in the medium containing $2.5 \mathrm{mM}$ glucose with or without 
treatment of selenite or glutamine deprivation for $24 \mathrm{~h}(\mathrm{~A})$ and $22 \mathrm{~h}(\mathrm{~B})$. C-D Cell viability of HCT116 cells cultured in the medium containing $2.5 \mathrm{mM}$ glucose with or without treatment of selenite or DFO or Fer-1 for $24 \mathrm{~h}$. E-F LPO levels (E) and LIP levels (F) of HCT116 cells cultured in the medium containing 25 or 2.5 $\mathrm{mM}$ glucose with or without treatment of selenite for $22 \mathrm{~h}$. Cells cultured in the medium containing 25 mM glucose were treated by 1 mM T-BHP or $200 \mu \mathrm{M}$ FAS for 30 min before detection. G-I Cyss levels (G), NADP+/NADPH ratios $(H)$ and GSH level $(\mathrm{I})$ of HCT116 cells cultured in the medium containing $2.5 \mathrm{mM}$ glucose with or without treatment of selenite or glutamine deprivation for $22 \mathrm{~h}$. Results are representative of three biologically independent experiments. Data are expressed as mean $\pm S D,{ }^{*} p<0.05,{ }^{\star \star} p<0.01$. GIn: glutamine, DFO: deferoxamine mesylate, Fer-1: ferrostatin-1, LPO: lipid peroxidation, LIP: labile iron pool $\triangle$ T-BHP: tert-butyl hydroperoxide, FAS: ferrous ammonium sulfate, Cyss: cystine, NADP+: oxidized form of nicotinamide-adenine dinucleotide phosphate, NADPH: reduced form of nicotinamide-adenine dinucleotide phosphate, GSH: glutathione. 
A HCT116 tumors

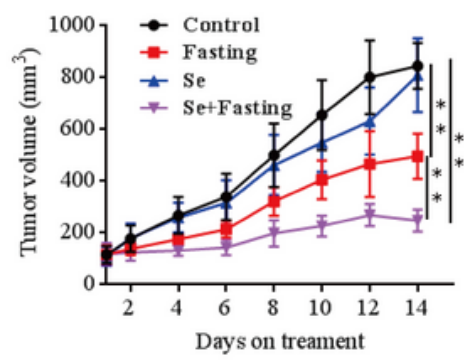

LLC tumors

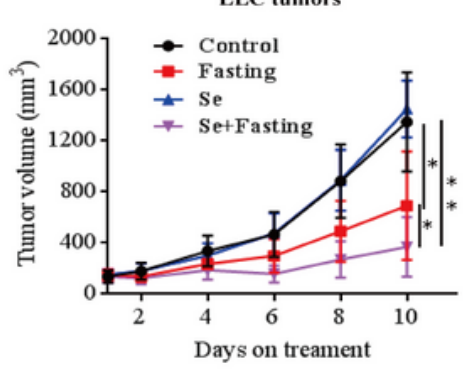

$\mathrm{C}$
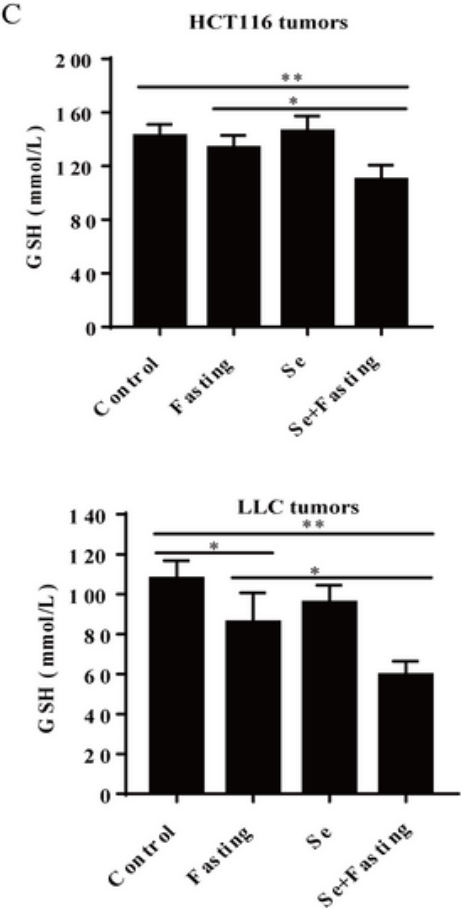

HCT116 tumors

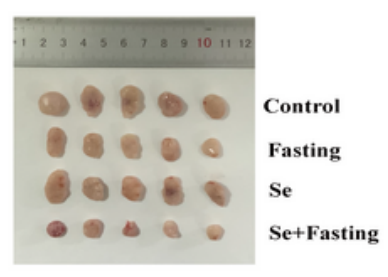

LLC tumors

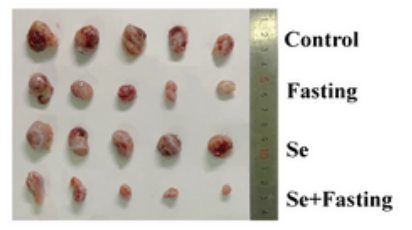

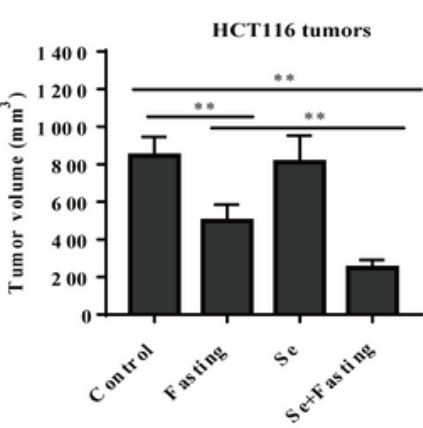

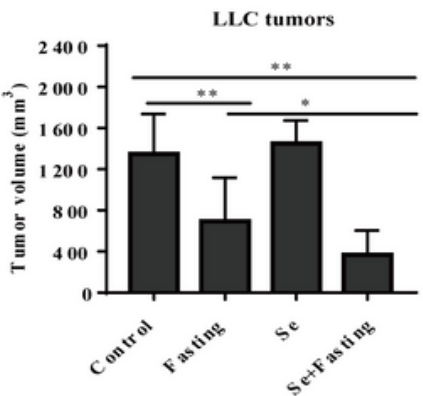

$\mathrm{E}$
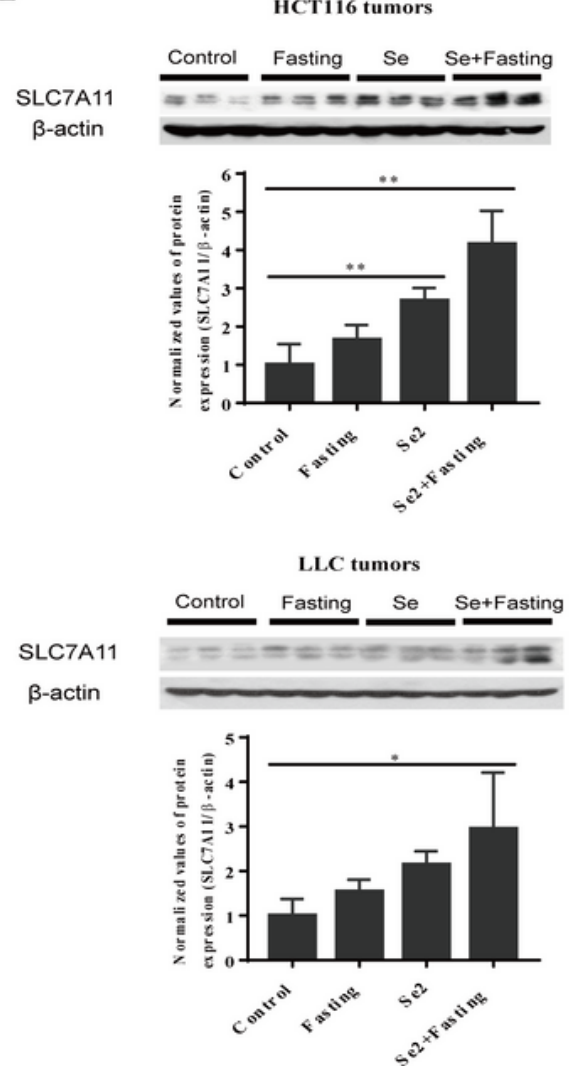

\section{Figure 6}

Fasting improves therapeutic efficacy of selenite in vivo. A In vivo growth of tumors as measured by tumor volume in mice inoculated with HCT116 cells or LLC cells, picture and volume of tumors isolated from mice in different groups. B Weight of tumors isolated from mice in different groups. C-E Measurement of GSH levels (C), NADP+/NADPH ratios (D) and SLC7A11 protein levels (E) of tumors isolated from mice in different groups. Data are expressed as mean $\pm S D$, * $p<0.05$, ** $p<0.01$. Control: 
control group, mice were fed ad libitum and treated with water, Fasting: fasting group, mice experienced feeding/fasting cycles for 24 hours, respectively, Se: selenite group, mice were fed ad libitum, and treated with $2 \mathrm{mg} / \mathrm{kg}$ body weight of selenite during fasting cycle, Se+Fasting: selenite-hypoglycemia combination group, mice experienced feeding/fasting cycles for 24 hours, respectively and were treated with $2 \mathrm{mg} / \mathrm{kg}$ body weight of selenite during fasting cycle, $\mathrm{n}=5$ per group. 